\title{
Energy Efficient Forwarding Scheme for Secure Wireless Ad Hoc Routing Protocols
}

\author{
Kwonseung Shin, Min Young Chung, and Hyunseung Choo* \\ School of Information and Communication Engineering \\ Sungkyunkwan University \\ 440-746, Suwon, Korea +82-31-290-7145 \\ \{manics86, mychung, choo\}@ece.skku.ac.kr
}

\begin{abstract}
Ad hoc networks can be used in scenarios such as military and emergency relief, and have the advantage of establishing communications independently. Securing the ad hoc routing protocol is necessary to prevent attacks in these applications. However, securing protocols for mobile ad hoc networks presents challenges due to energy consuming processes such as authentication of hosts and verification of messages. In this paper, we propose an efficient forwarding mechanism for secure ad hoc routing protocols, based on public key cryptography.
\end{abstract}

Keywords: Energy Efficiency, Public Key, and Secure Ad-hoc Routing.

\section{Introduction}

A mobile ad hoc network [1] is a set of wireless mobile hosts with the capability of cooperatively establishing communications independently. Due to this advantage, ad hoc networks can be used in military and emergency relief scenarios. In these and other ad hoc networking applications, security in the routing protocols is necessary to guard against attacks, such as malicious routing misdirection 2 . However, securing protocols for mobile ad hoc networks 3/4/5 presents challenges due to energy consuming processes such as authentication of hosts and verification of messages. Therefore, to extend the lifetime of wireless mobile ad hoc hosts with limited battery capacity, an energy efficient secure ad hoc routing protocol must be designed.

Most secure ad hoc routing protocols depending on the Certificate Authority (CA) provide authentication, non-repudiation, and integrity to prevent attacks such as modification, impersonation, and fabrication. Authenticated Routing for Ad Hoc Networks (ARAN) [6] is a secure ad hoc routing protocol using the CA. Initially each node receives a certificate, and public key of the CA, from the CA securely. In reactive routing protocols, when a source node wants to send a packet to a destination node, it broadcasts a route request packet if it has no route information about the destination node. However, in ARAN, the following route discovery process is performed to provide secure routing:

\footnotetext{
* Corresponding author.
} 
1. The source node signs a route request message using its private key and appends its own certificate. Then, it broadcast the message to all nodes within its radio frequency coverage area.

2. The one-hop neighbor of the source ignores the duplicate requests occurring when the RREQ is received multiple times. Otherwise, it verifies the certificate of the source by using the public key of the CA. Then it checks the signature of the source by using the public key extracted from the certificate of the source. If the verification is successful, the intermediate node set up a reverse path for the source, signs the RREQ, and appends its own certificate to the message. Then, it rebroadcasts the RREQ.

3. Intermediate nodes that receive the RREQ verify the source and node that has sent RREQ directly if the message has not already been received. Then, they use the public keys extracted from two certificates attached in the RREQ to validate the signatures. The intermediate nodes remove the signature and certificate of the node that sent RREQ directly if the verification is successful. Then, the nodes sign the message and rebroadcast it with their own certificate.

4. Until the RREQ message is flooded to all the nodes in the network, process 3 is repeated.

5. When the destination node eventually receives the RREQ, it creates a RREP and unicasts it toward the source node with its signature and certificate. The flooding process of RREP is similar to the processes 2 and 3 described above.

ARAN provides end-to-end security using cryptographic certificates, thereby preventing most attacks. However, appending the certificate each time each node sends route request packets, which is flooded to all nodes in the network, causes considerable network overhead. In this paper, we propose an efficient forwarding mechanism that reduces network overhead significantly by decreasing the frequency of included certificates.

\section{The Proposed Scheme}

In our scheme, each node that receives the route request packet that has a certificate verifies the certificate using the public key of the CA. Then, it stores the certificate to its own repository when the verification is successful. The sender does not include its certificate in messages when other nodes in the network have the certificate. On the other hand, it must include the certificate when it infers that other nodes in the network do not have its certificate. The decision of including certificate is made by states of nodes. Each node can be in on of two states, a Participation state or a Non-Participation state.

Fig. 1 represents the two states of nodes. The detailed rules regarding to the states are described as follows:

Non-Participation state: Nodes are initially in a Non-Participation state. When each node initiates a new route, it includes its certificate before broadcasting the route request message. Then, it enters the Participation state. A 
node sends a message with its certificate without changing state when the message is not a route request message.

Participation state: When a node enters the Participation state, it starts a timer $T_{B}$. The node changes to the Non-participation state by stopping $T_{B}$ when the $T_{B}$ expires. In the Participation state, a node does not include its certificate when it sends a message, because it infers that the other nodes in the network have its certificate. A node initiates timer $T_{B}$ when it broadcasts a route request message to initiate a new route. However, it does not initiate the timer when it broadcasts a route request message which is sent from other nodes.

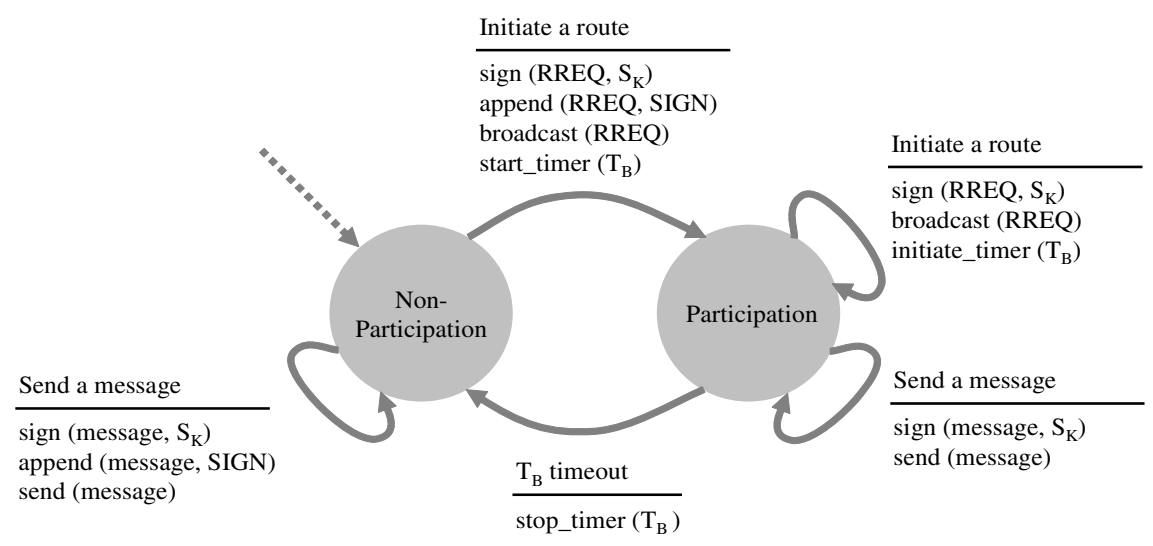

Fig. 1. The two states of nodes

Each node authenticates the sender using the public key of the CA and verifies the message using the public key included in the certificate when it receives the route request message that has a certificate from the other node. When the authentication and verification is successful, the node maintains the certificate in its repository. The certificate of a node in the repository expires when the route request message from the node is not received during $T_{M}$. Otherwise, the certificate of the node will be maintained permanently, though the node does not exist in the network. When a node receives a message that does not have certificates for authentication, it searches the certificate needed to authenticate the sender in its repository. When the certificate is found, it then verifies the signature of the received message without authentication of the sender, because the certificate in its repository has already been verified. Obviously, $T_{M}$ must be a larger value than $T_{B}$, and be set by considering the variation of propagation delay of route request messages that are caused by the movement of the node or the variation of network traffic.

Although $T_{M}$ is set properly, some nodes may receive the message with insufficient certificates for verification. For example, the node that newly participates in the network has no certificates of other nodes in the network. Therefore, the 
new node is not able to verify the sender or message when it receives a message that does not have complete set of certificates needed for verification. In this case, to obtain the certificates needed, the new node sends a Certificate REQuest (CREQ) message to neighbor node that has sent a message informing of insufficient certificates. The neighbor node is able to send certificates to the new node because it has verified the message successfully. The CREQ is signed using the private key of the sender, and the certificate is included only if the node is in the Non-Participation state. The node that receives the CREQ, verifies the sender and CREQ message. Then, it sends the Certificate REPly (CREP) message, including certificates requested by the CREQ. The CREP is also signed using a private key, and the certificate is included only if the sender of CREP is in the Participation state. Therefore, the new node that has no certificate is able to verify the message with insufficient certificates securely.

\section{Conclusion}

In this paper, we proposed an energy efficient forwarding scheme for secure ad hoc routing protocols using the CA. It reduces network overhead by decreasing the frequency of sending messages with certificates. By employing additional repository in each node, the energy efficiency of proposed scheme is increased significantly compared to conventional ARAN, when the nodes in the network initiate new routes frequently. In addition, the process of verification of hosts is also reduced, by eliminating the process of authentication that has already been performed.

Acknowledgments. This research was supported by the MIC(Ministry of Information and Communication), Korea, under the ITRC(Information Technology Research Center) support program supervised by the IITA(Institute of Information Technology Assessment), IITA-2006-(C1090-0603-0046)

\section{References}

1. Corson, S., Macker, J.: Mobile Ad hoc Networking (MANET): Routing Protocol Performance Issues and Evaluation Considerations. Network Working Group, Request for Comments: 2501. http://www.ietf.org/rfc/rfc2501.txt (1999)

2. Hu, Y-C., Perrig, A.: A survey of secure wireless ad hoc routing. Security \& Privacy Magazine, Vol. 2. IEEE (2004) 28-39

3. Papadimitratos, P., Haas, Z.J.: Secure Routing for Mobile Ad Hoc Networks. Proc. SCS Communication Networks and Distributed Systems Modeling and Simulation Conf. (2002)

4. Capkun, S., Hubaux, J.-P.: BISS: building secure routing out of an incomplete set of secure associations. Proc. 2nd ACM Wireless Security (2003) 21-29

5. Zapata, M.G., Asokan, N.: Securing Ad Hoc Routing Protocols. Proc. ACM Workshop on Wireless Security (2002) 1-10

6. Sanzgiri, K., LaFlamme, D., Dahill, B., Levine, B.N., Shields, C., Belding-Royer, E.M.: Authenticated Routing for Ad hoc Networks. Journal on Selected Areas in Communications, Vol. 23. IEEE (2005) 598-610 